\title{
OXIDATIVE ENZYMES AND THE INJURY METABOLISM OF DIAPAUSING CECROPIA SILKWORMS*
}

\author{
David G. Shappirio \\ Department of Zoology, The University of Michigan, Ann Arbor, Mich.
}

The remarkable control of insect growth by the hormone ecdyson is readily apparent in the events that transform a diapausing Cecropia pupa into a developing adult moth. The pupal diapause, as shown by Williams, ${ }^{1}$ is a state of developmental arrest enforced by the failure of the insect's endocrine system to supply the hormonal stimulus required for growth and development. In essence, what the individual diapausing tissues require is ecdyson, secreted by the prothoracic glands. The latter become inactive after pupation and remain so for many months; but when the prothoracic glands recover their secretory function under neuroendocrine stimulation from the brain, the individual tissues once again begin to grow, and commence a sequence of morphogenetic changes that culminates in three weeks' time with the emergence of the adult moth. ${ }^{\mathrm{I}}$

One of the principal tasks of present-day insect endocrinology is to seek an understanding of how ecdyson is able to exert its control over the growth process. In molecular terms, one avenue of approach to the problem lies in elucidating the biochemical changes that occur at the termination of diapause in response to the growth-promoting action of ecdyson. The properties of the diapausing insect itself are likewise of interest for understanding the physiological and biochemical adaptations of cells to life in a state of arrested development.

Both these matters were discussed by Williams ${ }^{2,3}$ about ten years ago in papers that called attention to the suitability of diapausing and developing silkmoths as subjects for examining biochemical mechanisms in insect growth and metamorphosis. At the time of these papers it was already clear that alterations in oxidative enzymes at the onset and termination of diapause had an important bearing on the problems just mentioned. In particular, and of special interest for the present paper, was the evidence that a synthesis of oxidative enzymes within the pupal tissues represented one type of change promoted by ecdyson in favoring growth. This matter has subsequently been explored in detail in a number of publications. ${ }^{4-8}$

In the present paper I propose to review recent progress in our understanding of respiratory metabolism and respiratory enzymes in relation to the diapause and development of the Cecropia silkworm. After a brief résumé of the metabolic and enzymatic changes during the course of metamorphosis, two principal topics will be considered: (1) the peculiar properties of respiratory metabolism in diapausing tissues and recent changes in our ideas as to their physiological basis; and (2) the close parallel between respiratory changes normally associated with the termination of diapause and those that follow

* The work described in this article was supported in part by research grants from the Faculty Research Fund of the Horace H. Rackham School of Graduate Studies, University of Michigan, Ann Arbor, Mich., and by Research Grant RG-6101 from the National Institutes of Health, Public Health Service, Bethesda, Md. 
localized integumental injury to diapausing pupae. Insight into the biochemical effects of integumental injury has considerably broadened our perspectives on the significance of respiratory changes, in relation to the hormonal control of diapause and development.

\section{Respiratory Metabolism and Metamorphosis}

It has been known for many years that the pupal stage in insect metamorphosis is characterized by a U-shaped curve as regards over-all respiratory metabolism. ${ }^{9}$ The descending limb of the curve follows pupation and a corresponding rise is correlated with adult development. This phenomenon is well exemplified in lepidopterous species that experience a pupal diapause. In the Cecropia silkworm, as shown by Schneiderman and Williams, ${ }^{10}$ the respiration rapidly falls after the pupal molt to a level less than two per cent of that of the same animal prior to pupation. Respiration persists at an extremely low level throughout diapause. Then, when growth and metamorphosis are resumed under the influence of ecdyson, respiration begins a

TABLE 1

Relative Cytochrome Concentrations in Individual Cecropia Tissues at Successive STAGES IN METAMORPHOSIS

\begin{tabular}{|c|c|c|c|c|}
\hline \multirow[t]{2}{*}{ Stage in metamorphosis } & \multicolumn{4}{|c|}{ Relative concentration of cytochrome* } \\
\hline & $b$ & $c$ & $a+a_{3}$ & $b_{5}$ \\
\hline $\begin{array}{l}\text { Larva } \\
\text { Diapausing pupa } \\
\text { Early developing adult } \\
\text { Late developing adult }\end{array}$ & $\begin{array}{l}++ \\
0 \\
+ \\
++\end{array}$ & $\begin{array}{l}++ \\
0 \\
+ \\
++\end{array}$ & $\begin{array}{c}+++ \\
+ \\
++ \\
+++\end{array}$ & $\begin{array}{l}t+t \\
t \\
t+ \\
t++\end{array}$ \\
\hline
\end{tabular}

* Relative concentrations indicated as +++ , high; ++ , moderate; + , low; 0 , not detectable. Observations at each stage based on several of the following: wing epidermis, fat body, heart, mid-gut, gonads, and malpighian tubules. Data from Shappirio and Williams.

rapid rise that continues during the formation of the adult moth. In concurrent studies, Schneiderman and Williams ${ }^{5}$ also established in Cecropia that the scant respiration of the diapausing pupa is relatively insensitive to cyanide and carbon monoxide, in concentrations that markedly suppress the respiration of the pre- and postdiapausing insect.

\section{The Cytochrome System and Metamorphosis}

Additional insight into these matters has been provided subsequently through studies of the respiratory enzymes themselves. By spectroscopic methods, based on the low-temperature technique of Keilin and Hartree, ${ }^{11}$ it was possible to characterize conspicuous alterations in the cytochrome system of individual Cecropia tissues during the course of metamorphosis. ${ }^{7}$ These observations are illustrated in TABLF, 1 , which summarizes changes occurring in epidermis, fat body, heart, mid-gut, gonads, and malpighian tubules. As shown in TABLE 1 , prior to the pupal diapause the rapidly growing larval tissues contain moderate to high concentrations of cytochromes $b, c, a+a_{3}$, and $b_{5}$. In contrast, the tissues of the diapausing pupa show a peculiar cytochrome 
system in that components $b$ and $c$ are not detectable spectroscopically. Cytochromes $a+a_{3}$ and $b_{5}$ persist in low, although detectable, concentration. Several months later, when growth and metamorphosis are resumed in response to ecdyson, cytochromes $b$ and $c$ reappear in detectable concentration. Together with $a+a_{3}$ and $b_{5}$ they then undergo a progressive increase in concentration during the course of adult development.

It is worth noting at this point that the intersegmental muscles of the insect retain a normal cytochrome system, containing moderate titers of components $b, c$, and $a+a_{3}$ throughout diapause, as well as in the pre- and postdiapausing insect. Apparently the somatic muscles do not participate in the changes just described. Recent studies (Shappirio, unpublished) show that the pupal brain also retains detectable concentrations of cytochromes $b, c$, and $a+a_{3}$ throughout diapause. Information on the status of the cytochrome system in the pupal brain has not hitherto been available.

However, for epidermis, fat body, heart, gut, gonads, and malpighian tubules, one can summarize the spectroscopic studies as giving evidence for a marked breakdown of cytochromes after pupation and an equally prominent resynthesis associated with the termination of diapause. These conclusions have been strengthened greatly by the finding ${ }^{8}$ that several oxidative enzyme systems, including DPNH oxidase, DPNH-cytochrome $c$ reductase, succinatecytochrome $c$ reductase, and cytochrome $c$ oxidase, show alterations in activity, coupled with the onset and termination of diapause, which essentially parallel the spectroscopic changes described above.

Let us consider the implications of these conclusions in relation to the respiratory metabolism of the diapausing insect. The best way to view cellular respiration is in terms of its role as a chemical energy transformer to provide high energy phosphorus and sulfur compounds for meeting the endergonic demands of living cells. Pupal respiration is exceedingly low; but the Cecropia pupa is nevertheless an obligatorily aerobic organism that depends on respiration in order to exist. ${ }^{12}$ In the absence of extensive muscular activity we can probably generalize and say that the principal energy requirements of the pupa are for. synthetic reactions and active transport processes. In relation to the former, several workers have shown that the incorporation of labeled precursors into proteins and nucleic acids, and presumably their synthesis, continue during diapause but at a low level. ${ }^{13-15}$ If one regards cytochrome concentrations as reflecting the metabolic requirements of the tissue, ${ }^{16}$ then the characteristics of the cytochrome system in pupal tissues can be viewed as an adaptation to life in a state of arrested development.

\section{Terminal Oxidative Melabolism in Diapausing Tissues}

The over-all respiration of the diapausing pupa is interesting not only because of its extremely low rate, but also because of its relative insensitivity to inhibitors of cytochrome oxidase such as carbon monoxide. ${ }^{5}$ When the latter inhibits respiration, and particularly if this inhibition is reversed by light, one may conclude with reasonable certainty that the respiration is mediated via the classical cytochrome $c$ oxidase pathway $\left(a+a_{3}\right)$. However, when carbon monoxide fails to show a large inhibitory effect even at relatively 
high $\mathrm{CO} / \mathrm{O}_{2}$ ratios, a conclusion regarding the terminal oxidative pathway is commonly rendered much more difficult. Such has been the case with diapausing silkmoth pupae, as with a variety of plant and animal tissues showing sensitivity to the inhibitor.

Insensitivity to carbon monoxide would arise if respiration were mediated by a terminal oxidase, other than $a+a_{3}$, and capable of functioning in the presence of the inhibitor. Some years ago it appeared that the CO-resistant properties of pupal respiration could be best explained in terms of such a different terminal oxidative pathway. ${ }^{5}$ 'The most promising candidates for the role of terminal oxidase appeared to be an autoxidizable flavoprotein or cytochrome.

More recently, however, experimental evidence has been obtained ${ }^{17,18,31}$ in favor of an alternative explanation of pupal respiratory metabolism: namely, that the respiration is mediated by cytochrome oxidase itself. This explanation rests on the theory that cytochrome oxidase-mediated respiration becomes insensitive to carbon monoxide under certain conditions. These conditions demand, principally, that oxidase be present in large excess in relation to the actual rate of electron transport (that is, respiratory activity). Under such conditions the oxidase may be regarded as relatively "unsaturated" by electron transfer; even at high concentrations of carbon monoxide, the equilibrium between oxidase and inhibitor probably affords sufficient free oxidase to satisfy the relatively low respiratory requirements.

Although evidence for this theory has been available for a number of years, ${ }^{19,20}$ its applicability in the case of diapausing Cecropia pupae became apparent only relatively recently ${ }^{8}$ upon the realization that conditions in most pupal tissues were probably of just the right sort to permit the function of cytochrome $a_{3}$ as an "insensitive" oxidase. As will be recalled, in most pupal tissues cytochromes $a+a_{3}$ are present while cytochromes $b$ and $c$ are below a detectable concentration. Moreover, the respiration is extremely low.

The principal published evidence on diapausing pupae is that of Harvey and Williams ${ }^{17}$ for the pupal heartbeat and that of Kurland and Schneiderman ${ }^{18}$ for the pupa as a whole. As shown by these workers, heartbeat and respiration are not affected by lowering the partial pressure of oxygen to that of a few millimeters of mercury. But under these conditions both processes become sensitive to carbon monoxide. At the low oxygen pressure, turnover of cytochrome oxidase is presumably rate-limiting, and the great excess of oxidase is removed. These experiments provide a persuasive argument that pupal heartbeat and respiration are mediated by cytochromes $a+a_{3}$ and not by some alternative oxidase.

If cytochrome oxidase is present and functioning in the diapausing tissues, then one is obliged to inquire as to the whereabouts of cytochrome $c$, which is undetectable spectroscopically. ${ }^{7}$ Since cytochrome $c$ is the only known substrate for cytochrome oxidase, at least in vivo, the simplest explanation is that $c$ is actually present in the pupal tissues, but not in detectable concentration.

This analysis of respiratory metabolism in diapausing tissues leads to several important conclusions. In the first place, it signifies that the CO-resistance of the pupa conceals a fundamental dependence of respiration on cytochrome oxidase. Since the pre- and postdiapausing tissues likewise depend on cyto- 
chrome oxidase-mediated respiration, it persuades one to conclude ${ }^{18}$ that the respiratory metabolism shows quantitative variations during metamorphosis, but not qualitative changes as had hitherto seemed probable. ${ }^{5}$

One would also predict, on the basis of the analysis described above, that the primary determinant for sensitivity to carbon monoxide is the magnitude of respiratory electron transfer in relation to the potential turnover of the available cytochrome oxidase. When the subterminal electron transfer proceeds rapidly and substantially saturates the oxidase, sensitivity is high. Otherwise the effect of carbon monoxide is low, even though cytochrome oxidase mediates the respiration that occurs. This analysis is supported particularly well in studies, to be described later in this paper, dealing with the CO-sensitivity of pupae whose respiratory metabolism is accelerated after integumental injury. ${ }^{18,31}$

\section{Respiratory Metabolism at the Termination of Diapause}

We may now return to the events that occur when pupal diapause is terminated, in response to the action of ecdyson. At this time, cytochromes $b$ and $c$ reappear in spectroscopically detectable titer within the individual tissues, and together with $a+a_{3}$ and $b_{5}$ commence a rise in concentration that accompanies adult development. Actually the onset of these changes can be detected several days prior to the first morphological sign of development in terms of increased activity of several oxidative enzyme systems. ${ }^{8}$

Simultaneously the respiratory metabolism begins a conspicuous rise and becomes progressively more sensitive to carbon monoxide. ${ }^{5,10}$ According to present views, and as stated by Kurland and Schneiderman, ${ }^{18}$ the increased sensitivity reflects a correspondingly enhanced saturation of cytochrome oxidase.

Schneiderman and Williams have also shown ${ }^{5}$ that when carbon monoxide is applied to pupae just prior to the outset of adult development, respiration fails to rise and the insect remains in diapause. And if the gas is applied to early postdiapausing animals, during the first few days of adult development, respiration is suppressed to about the diapausing level. Developmental progress then ceases. These experiments show that function of the normal cytochrome oxidase system, at rates above that in the diapausing pupa, is essential for the termination of diapause. One can conclude that cytochrome synthesis, and the enhanced respiratory metabolism that it permits, are prerequisites for the insect's developmental response to ecdyson. ${ }^{5}$

In the normal course of events, cytochrome synthesis is inextricably associated with adult development, and it is difficult to determine how closely the cytochrome synthesis is related to the primary action of ecdyson. As has been stated elsewhere, the changes in the cytochrome system at the termination of diapause may be typical of a number of synthetic processes, all required for development, but which occur following some more fundamental action of ecdyson upon the diapausing cell. ${ }^{15,21}$ In order to resolve this question, one might seek a means of dissociating the cytochrome synthesis from the termination of pupal diapause and the developmental response. Such an opportunity has to a large extent been provided by studies on the respiratory enzymes of pupae receiving localized integumental injury. 


\section{Injury Melabolism}

During the course of their metabolic studies, Schneiderman and Williams ${ }^{10}$ confirmed for Cecropia that localized injuries cause a conspicuous rise in the animal's respiration. This enhanced respiratory metabolism is termed the "injury metabolism." The magnitude and duration of the injury metabolism were found to depend on the extent of injury. Large injuries, involving the removal of sizable areas of pupal integument, cause a rise in respiration that continues for several days, until the metabolism equals or exceeds that of the postdiapausing insect. Respiration remains at the high level for many weeks and then gradually declines, reaching the initial level only after several months.

It is of special interest and importance to note that although metabolism attains a pace that equals or exceeds that when diapause is terminated, injury is followed by no over-all development and, in fact, no morphogenetic response save for a healing of the epidermal wound. ${ }^{10}$ This healing response is localized; yet the metabolic changes of the animal are far too large to be accounted for solely in terms of an activated metabolism in the localized area of wounding.

The injury metabolism is characteristic only of diapausing pupac. ${ }^{12}$ Considerable evidence argues that it is not attributable to muscular activity and that the metabolic response does not depend on the insect's central nervous system..$^{10,12}$ Moreover the injury metabolism can be obtained in isolated pupal abdomens devoid of the known endocrine organs. ${ }^{10,12}$ It appears to be generalized metabolic response on the part of the individual diapausing tissues.

The effect of carbon monoxide on the injury-stimulated respiration is a matter of particular interest. According to the hypothesis presented earlier, the degree of sensitivity to this inhibitor will rise as increasing respiratory rate provides a greater "saturation" of cytochrome oxidase. Thus, diapausing pupae receiving only very small injuries, and showing only a relatively slight increase in respiratory metabolism, would be expected to show a sensitivity to carbon monoxide only slightly greater than in the case of uninjured pupae. Large injuries, on the other hand, should lead to a greatly increased sensitivity, in keeping with the greater enhancement of respiratory rate. On the basis of considerable experimental evidence, treated elsewhere, ${ }^{24,18,31}$ it is possible to conclude that the above view is substantially correct. Sensitivity to carbon monoxide is a function of respiratory rate.

The latter conclusion applies not only to injured pupae, but, according to this evidence, to uninjured pupae and to developing adults. On the basis of inhibitory studies, one is drawn ever closer to the view that respiration in Cecropia shows a fundamental dependence on cytochrome oxidase throughout pupal diapause and adult development.

For the pupa as a whole, the alterations in respiratory metabolism after injury strikingly parallel those that accompany the termination of diapause. Therefore it has been a matter of considerable importance to determine whether the over-all metabolic effects of injury are accompanied by alterations in the cytochrome system in individual tissues. We may now consider several series of experiments designed to provide an insight into this question. A preliminary account of these experiments has already been published, ${ }^{22}$ and they will be considered in detail elsewhere. ${ }^{31}$ The studies are based on diapausing pupae 
in which a large integumental injury was established by excising cuticle and epidermis from the facial and leg region. Routine surgical techniques, developed by Williams for silkmoth pupae, were utilized; and the wounded area was covered by a previously shaped piece of cellulose acetate sealed in place with melted paraffin wax. ${ }^{23}$

Such an injury is routinely followed, as shown in FIGURE 1, by an immediate doubling of respiration and a subsequent rise that persists for several days. In the case of the pupa shown, the respiration leveled off at about seven times that measured prior to the injury. The metabolic rate of animals receiving

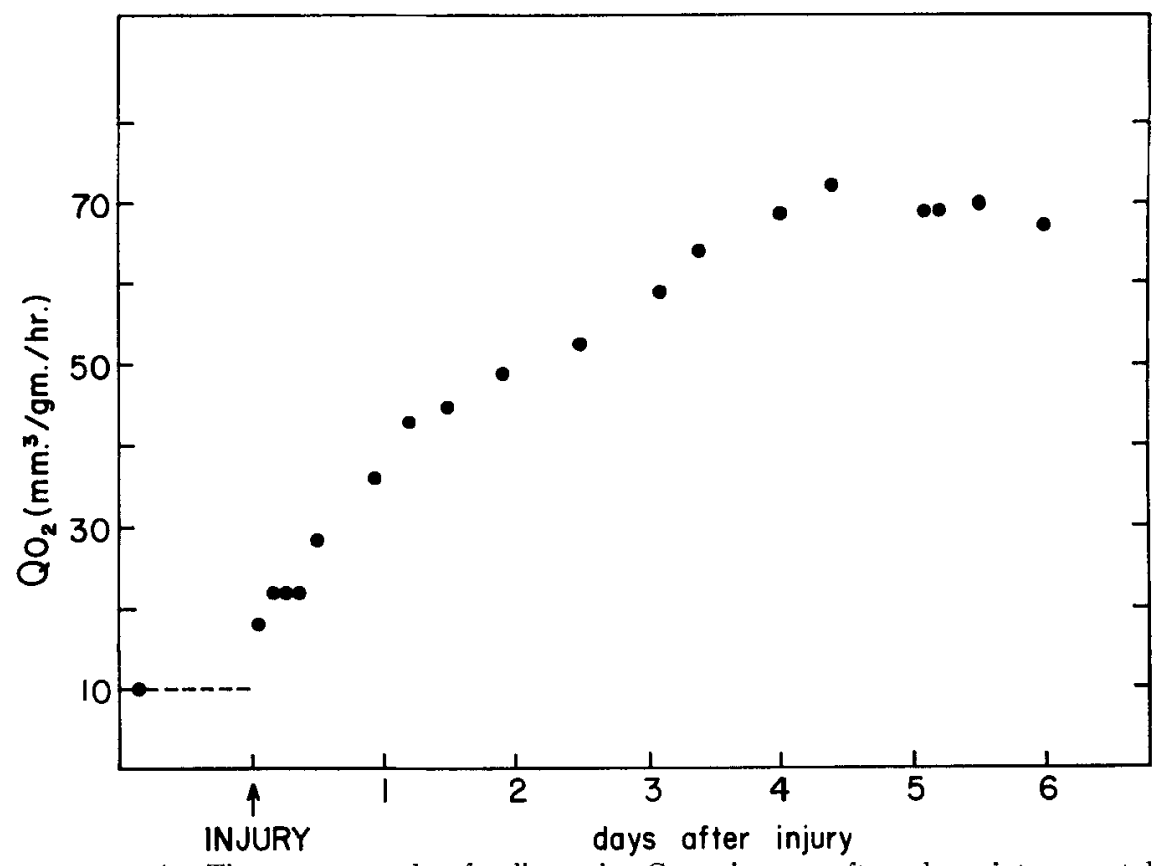

Figure 1. The oxygen uptake of a diapausing Cecropia pupa after a large integumental injury. The injury involved removal of cuticle and epidermis in the facial and leg region.

the large injury is quantitatively equivalent to that of animals at the outset of adult development.

Principal efforts were directed toward a single tissue, the wing epithelium; and to the measurement of several oxidative enzyme systems, including DPNH oxidase, DPNH-cytochrome $c$ reductase, succinate-cytochrome $c$ reductase, and cytochrome $c$ oxidase. Considerable information on the behavior of these enzyme systems in wing homogenates was already available; measurements of their activities at successive stages in pupation, pupal diapause and adult development had been utilized $^{8}$ to provide quantitative confirmation of the spectroscopic observations discussed earlier.

TABLE 2 permits one to observe the effect of large integumental injury on the activities of oxidative enzyme systems in the wing. The upper horizontal row of figures shows activities encountered in wing preparations from uninjured 
diapausing pupae. In the second row of figures are illustrated the activities in wings of pupae from the same batch of diapausing animals, but prepared for analysis one week after injury. If one compares the uninjured and injured pupae, it is readily apparent that the latter reveal a detectable level of DPNH oxidase activity whereas this activity is not detectable in wings prior to injury. The appearance of detectable DPNH oxidase activity involves more than a twofold increase in activity as expressed on a nitrogen basis. DPNH-cytochrome $c$ reductase, succinate-cytochrome $c$ reductase, and cytochrome $c$ oxidase likewise show a two- to threefold increase in activity following the injury.

The levels of oxidative activity encountered in wings from injured pupac are of the same order of magnitude as those observed in animals just after the termination of pupal diapause. This will be evident from the third line of

TABle 2

Fafects of Integumental Injury on the Activity of Oxidative Enzyme Systems IN THE WING EPITHELIUM

\begin{tabular}{l|c|c|c|c|c}
\hline \multicolumn{1}{c|}{ Type of animal } & $\begin{array}{c}\text { Number of } \\
\text { experiments }\end{array}$ & $\begin{array}{c}\text { DPNH } \\
\text { oxidase }\end{array}$ & $\begin{array}{c}\text { DPNH-cyto- } \\
\text { chrome } c \\
\text { reductase }\end{array}$ & $\begin{array}{c}\text { Succinate- } \\
\text { cytochrome } c \\
\text { reductase }\end{array}$ & $\begin{array}{c}\text { Cytochrome } \\
\text { coxidase }\end{array}$ \\
\hline $\begin{array}{l}\text { Uninjured diapausing pupae } \\
\text { Pupae one week after injury }\end{array}$ & 6 & $<3$ & 66 & 24 & 300 \\
$\begin{array}{l}\text { Animals at outset of adult de- } \\
\text { velopment }\end{array}$ & 4 & 11 & 114 & 48 & 1500 \\
Pupae six months after injury & 3 & $<5$ & 80 & 18 & 290 \\
\hline
\end{tabular}

* In each experiment, the wings from six insects were pooled, and then homogenized and prepared by differential centrifugation, as described elsewhere, ${ }^{8}$ to yield a combined mitochondrial-microsomal suspension for spectrophotometric assay of each of the enzyme systems. The activities are averages for all experiments, and are expressed on a nitrogen basis, namely: DPNH oxidase, as millimicromoles DPNH oxidized $/ \mathrm{mg}$. N/min.; DPNH- and succinatecytochrome $c$ reductase, as millimicromoles cytochrome $c$ reduced $/ \mathrm{mg}$. N/min.; and cyto. chrome $c$ oxidase, as millimicromoles cytochrome $c$ oxidized $/ \mathrm{mg}$. N/min. When DPNH oxidase activity was below a detectable level, the table records "less than" the minimum activity that would have produced a detectable change in absorption.

TABIE 2, which illustrates activities in wings from animals on the "zero" to second day of adult development.* Except possibly in the case of cytochrome $c$ oxidase, it is essentially impossible to distinguish between wings from injured pupae and those from animals at the outset of adult development.

Six months after injury, pupae belonging to the same batch that was utilized earlier had recovered from the injury metabolism, in that their respirations had declined to levels characteristic of uninjured diapausing pupae. TABLE 2 illustrates that oxidative activities in wing preparations also declined. DPNH oxidase activity was found to be undetectable, and the other enzyme systems exhibited activities substantially similar to those encountered prior to injury. This "recovery" of oxidative enzyme systems is summarized graphically in FIGURE 2, which illustrates DPNH-cytochrome $c$ reductase, succinate-cytochrome $c$ reductase, and cytochrome $c$ oxidase activities plotted relative to the preinjury level for uninjured, injured, and "recovered" individuals. In the

* Stages in adult development were ascertained according to criteria of Schneiderman and Williams. ${ }^{6}$ 
case of each enzyme system, one can conclude that injury causes a two- to threefold increase in activity within a week's time, and that the activities subsequently decline to about the preinjury level.

The above experiments reveal a doubling or tripling in enzymatic activities one week after injury, when the activities are calculated on a nitrogen basis. In actual fact, the nitrogen content of the wing preparations itself shows an approximate doubling after injury. Consequently the actual increase in absolute activity is four- to sixfold. In the present case it seems likely that this increase in activity is associated at least in part with an actual synthesis of oxidative enzymes within the wing. By spectroscopic means, one can observe an increased concentration of cytochromes $b_{5}$ and $a+a_{3}$ in wings from injured pupae, and in favorable preparations it is also possible to observe a detectable absorption band of cytochrome $c$. Unfortunately, the technique of low-temperature visual spectroscopy utilized does not afford a quantitative insight into the magnitude of the increase in cytochrome concentration.

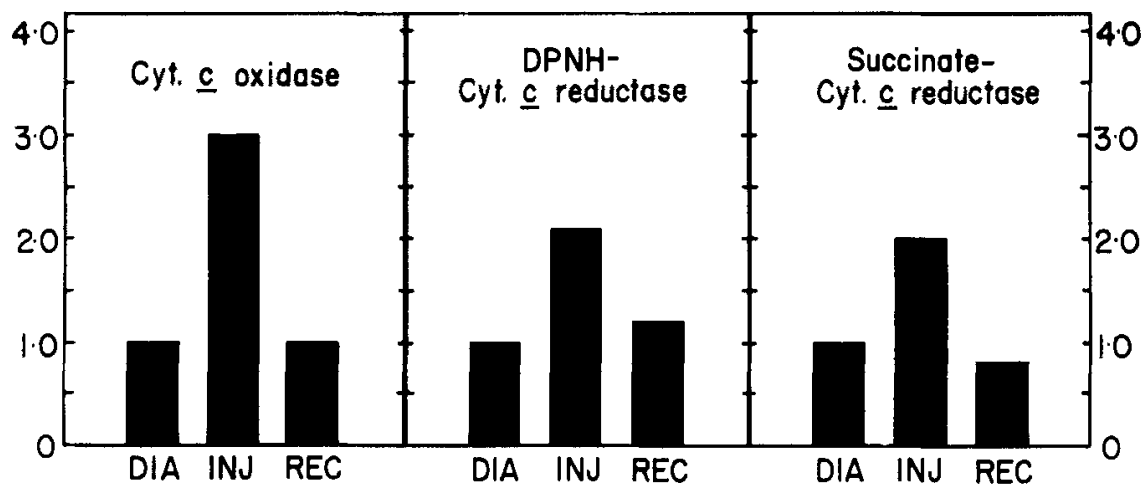

FIGURE 2. Activities of oxidative enzyme systems in uninjured diapausing pupae (DIA), pupae one week after injury (INJ), and "recovered" pupae six months after injury (REC). The activities are shown relative to the preinjury level. Data from TABLE 2.

A body of evidence is now accumulating that suggests that the oxidative changes just described are accompanied by equivalent enhancement of a variety of endergonic processes within the pupa. It has been known for some years from the work of Telfer and Williams ${ }^{13,14}$ that the rate of incorporation of $\mathrm{C}^{14}$-labeled amino acids into pupal blood proteins is low, but that it is augmented after injury by about the same extent as the increase in respiratory metabolism. And in the wing itself, G. R. Wyatt (personal communication) has observed that the incorporation of radioactive phosphate into ribonucleic acid is also enhanced five- or sixfold after injury.

It is important to emphasize that these biochemical changes parallel those normally occurring when diapause is terminated. Indeed, on the basis of the biochemical criteria thus far studied in Cecropia, one is virtually unable to distinguish injured pupae from animals at the outset of adult development.

However, as stated earlier, the injured pupa fails to reveal any of the morphogenetic changes that characterize the onset of adult development in response to ecdyson. The wings of injured pupae do not begin to differentiate; 
nor do the spermatogonia or spermalocytes within testes of male pupae show any visible changes after injury, as demonstrated by Williams (personal communication), although their prominent differentiation into spermatozoa is one of the earliest signs of adult development. It seems necessary to conclude that cytochrome synthesis and accelerated incorporation of precursors into pupal protein and nucleic acid are not sufficient in themselves to permit the termination of diapause.

\section{Injury, Wound-healing, and Hormones}

The only morphogenetic response thus far observed in injured Cecropia pupae is localized: a healing of the epidermal wound. Certain information about the wound-healing process is already available. ${ }^{25-27}$ Under the plastic window the wound is first healed by a thin membrane formed from blood cells that migrate to the wounded site; the area is then invaded by ingrowing epidermal cells and tracheoles that derive from the surrounding tissue. According to Smith and Schneiderman, ${ }^{27}$ the healing process is unaccompanied by mitotic activity. Schneiderman ${ }^{6}$ has also demonstrated that wound-healing is prevented by exposure of the animal to carbon monoxide, or by prior injection of diphtheria toxin; these experiments show that the wound repair in itself depends on oxidative metabolism and imply that cytochrome synthesis is required.

Healing of epidermal wounds is, presumably, a desirable process for the pupa. But what is of particular interest in the present context is that seemingly the biochemical resources of the animal as a whole are mobilized in response to localized injury. Apparently in Cecropia an "injury factor," released from the wounded site, is of importance in evoking the generalized metabolic response; injury to one member of a pair of pupae joined in parabiosis causes an injury metabolism in the other pupa..$^{12,28}$

Insect endocrinologists will recognize a familiar concept in this idea of an "injury factor," and, more particularly, in the similarity between events occurring after injury and those that normally accompany growth. As long ago as 1937 , Wigglesworth ${ }^{29}$ pointed out that the localized changes following small epidermal injuries to Rhodnius show a striking similarity to events in the early stages of normal growth and molting. Wigglesworth concluded that a diffusible factor, perhaps a product of autolysis in the injured region, somehow activated the surrounding cells. He has subsequently ${ }^{30}$ continued a detailed cylological study of wound repair in Rhodnius. Wigglesworth makes it quite clear that the changes after injury proceed in the absence or inactivity of the brain and thoracic glands. He re-emphasizes that the injury factor has the same effect as ecdyson in the localized area of wounding; and the possibility remains open that the factor may even be ecdyson itself. The latter conjecture is, of course, exceptionally intriguing, since it implies that tissues other than the thoracic glands may be capable of producing ecdyson and that the endocrine function of these organs represents the specialized production of, as expressed by Wigglesworth, "some widespread metabolite." However, in so far as the Cecropia silkworm is concerned, we have no evidence for the presence of ecdyson, at least in concentrations capable of eliciting a morphogenetically detectable response in the direction of adult development. 


\section{Conclusions}

Studies on the injury metabolism of diapausing silkworms have provided us with a clearer perspective on several important matters. First, they have helped provide an insight into the probable basis of the respiratory insensitivity to carbon monoxide that characterizes diapausing pupae. According to present views, respiration is mediated by cytochrome oxidase, and the degree of saturation of the oxidase by subterminal electron transport is a prime determinant of sensitivity to the inhibitor. This permits us to conclude that despite major alterations in respiratory metabolism and sensitivity to inhibitors, respiration of the Cecropia silkworm depends fundamentally on cytochrome oxidase at all stages of the life history. Studies of injury metabolism also permit us to draw certain conclusions regarding the relationship between cytochrome synthesis and the action of ecdyson in terminating pupal diapause. As we have already seen, it appears that this synthesis, although a prerequisite for adult development, is not in itself sufficient to permit the initiation of adult development. It is clear that growth and metamorphosis require energy supplies above and beyond the requirements for maintenance of the diapausing state. But the provision of this energy supply is not enough to evoke growth and metamorphosis.

\section{Acknowledgments}

I thank C. M. Williams and W. R. Harvey for many helpful discussions. I am also indebted to C. M. Williams and G. R. Wyatt for their courtesy in permitting reference to unpublished experiments.

\section{References}

1. Williams, C. M. 1952. Biol. Bull. 103: 120-138.

2. Williams, C. M. 1948. Growth. 12 (Suppl.): 61-74.

3. Williams, C. M. 1951. Federation Proc. 10: 546-552.

4. Pappenheimer, A. M., Jr. \& C. M. Williams. 1952, J. Gen. Physiol. 35: 727-740.

5. Schneiderman, H. A. \& C. M. Williams. 1954. Biol. Bull. 106: 210-229.

6. Schnemerman, H. A. \& C. M. Williams. 1954. Biol. Bull. 106: 238-252.

7. Shappirio, D. G. \& C. M. Williams. 1957. Proc. Roy. Soc. London. B147: $218-232$.

8. Shappirio, D. G. \& C. M. Williams. 1957. Proc. Roy. Soc. London. B147: 233-246.

9. Heller, J. 1926. Biochem. Z. 169: 208-234.

10. Schneiderman, H. A. \& C. M. Willlams. 1953. Biol. Bull. 105: 320-334.

11. Keilin, D. \& E. F. Hartree. 1949. Nature. 164: 254.

12. Schnerderman, H. A. 1957. In Physiological Triggers. : 46-59. T. H. Bullock, Fel. Ronald Press. New York, N.Y.

13. Telfer, W. H. \& C. M. Williams. 1955. Anat. Record. 122: 441-442.

14. Telfer, W. H. \& C. M. Williams. J. Insect Physiol. In press.

15. WyatT, G. R. 1958. In The Chemical Basis of Development. : 807-811. W. D. McElroy \& B. Glass, Eds. Johns Hopkins Press. Baltimore, Md.

16. Drabkin, D. R. 1955. In Ciba Foundation Symposium on Porphyrin Biosynthesis and Metabolism. :96-124. G. E. W. Wolstenholme and E. C. P. Millar, Eds. Little, Brown \& Co. Boston, Mass.

17. Harvey, W. R. \& C. M. Williams. 1958. Biol. Bull. 114: 36-53.

18. Kurland, C. G. \& H. A. Schneiderman. 1959. Biol. Bull. 116: 136-161.

19. Hill, R. \& E. F. Hartree. 1953. Ann. Rev. Plant Physiol. 4: 115-150.

20. Keilin, D. \& F. F. Hartree. 1953. Nature. 171: 413-416.

21. Wigglesworth, V. B. 1954. The Physiology of Insect Metamorphosis. Cambridge Monographs in Experimental Biology. Cambridge Univ. Press. Cambridge, England.

22. Shappirio, D. G. 1958. Anat. Record. 132: 506.

23. Williams, C. M. 1959. Biol. Bull. 116: 323-338.

24. Harvey, W. R. 1956. Anat. Record. 126: 556. 
25. Williams, C. M. 1946. Biol. Bull. 90: 234-243.

26. WiJ.LiAMs, C. M. 1947. Biol. Bull. 93: 89-98.

27. Smith, R. D. \& H. A. Schnemerman. 1954. Anat. Record. 120 (3).

28. Harvey, W. R. 1957. Metabolic Studies on the Cecropia Silkworm, with Special Reference to the Pupal Diapause. Doctoral Dissertation. Harvard Univ. Library. Cambridge, Mass.

29. WiggLESWORTH, V. B. 1937. J. Exptl. Biol. 14: 364-381.

30. Wigglesworti, V. B. 1957. In Symposia of the Society for Experimental Biology. XI. The Biological Action of Growth Substances. : 204-227. Cambridge Univ. Press. Cambridge, England.

31. Harvey, W. R. \& D. G. Shappirio. To be published. 